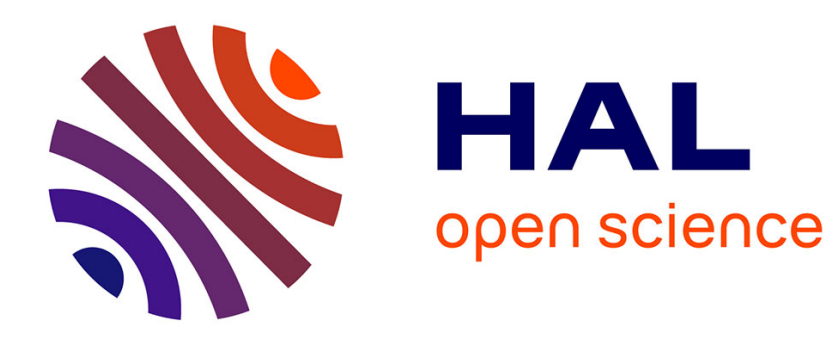

\title{
Dire et taire : l'intersubjectivité dans l'entretien de recherche
}

\author{
Estelle Masson, Valérie Haas
}

\section{To cite this version:}

Estelle Masson, Valérie Haas. Dire et taire : l'intersubjectivité dans l'entretien de recherche. Bulletin de psychologie, 2010, 63 (1), pp.5-13. 10.3917/bupsy.505.0005 . hal-01880660

\section{HAL Id: hal-01880660 \\ https://univ-rennes2.hal.science/hal-01880660}

Submitted on 25 Sep 2018

HAL is a multi-disciplinary open access archive for the deposit and dissemination of scientific research documents, whether they are published or not. The documents may come from teaching and research institutions in France or abroad, or from public or private research centers.
L'archive ouverte pluridisciplinaire HAL, est destinée au dépôt et à la diffusion de documents scientifiques de niveau recherche, publiés ou non, émanant des établissements d'enseignement et de recherche français ou étrangers, des laboratoires publics ou privés. 


\section{DIRE ET TAIRE : L'INTERSUBJECTIVITÉ DANS L'ENTRETIEN DE RECHERCHE}

Estelle Masson, Valérie Haas

Groupe d'études de psychologie | «Bulletin de psychologie »

2010/1 Numéro 505 | pages 5 à 13

ISSN 0007-4403

Article disponible en ligne à l'adresse :

https://www.cairn.info/revue-bulletin-de-psychologie-2010-1-page-5.htm

\section{Pour citer cet article :}

Estelle Masson, Valérie Haas « Dire et taire : l'intersubjectivité dans l'entretien de recherche », Bulletin de psychologie 2010/1 (Numéro 505), p. 5-13.

DOI 10.3917/bupsy.505.0005

Distribution électronique Cairn.info pour Groupe d'études de psychologie.

(c) Groupe d'études de psychologie. Tous droits réservés pour tous pays.

La reproduction ou représentation de cet article, notamment par photocopie, n'est autorisée que dans les limites des conditions générales d'utilisation du site ou, le cas échéant, des conditions générales de la licence souscrite par votre établissement. Toute autre reproduction ou représentation, en tout ou partie, sous quelque forme et de quelque manière que ce soit, est interdite sauf accord préalable et écrit de l'éditeur, en dehors des cas prévus par la législation en vigueur en France. Il est précisé que son stockage dans une base de données est également interdit. 


\author{
Masson Estelle* \\ HAAS Valérie**
}

\section{Dire et taire : l'intersubjectivité dans l'entretien de recherche}

Dans le cadre de la recherche en psychologie sociale, le recours aux méthodes qualitatives, en général, et à l'entretien non directif, en particulier, se révèle particulièrement précieux pour le chercheur qui cherche à connaître les mécanismes des représentations sociales et à saisir le sens subjectif, que recouvrent ces dernières pour le sujet. En effet, l'intérêt majeur des entretiens non directifs réside dans le fait que le chercheur n'impose pas au sujet un pré-découpage de l'objet, ce qui rend possible une approche holistique du phénomène étudié et des significations qu'il recouvre. Cela permet, bien souvent, de saisir l'existence, chez un même sujet, de modes d'appréhension pluriels d'un même objet, renvoyant au processus de polyphasie cognitive (Haas, 2006). Cependant, comme nous le verrons ici, la situation d'entretien relève d'une situation d'interaction sociale, où se joue « un jeu à trois pôles » (Kauffmann, 1996), entre un interviewer, un interviewé et un objet, dont le déroulement détermine les productions narratives des sujets et, par conséquent, les contenus dont disposera le chercheur pour son analyse.

S'intéresser à la situation d'entretien, au travers du prisme du regard psychosocial, c'est-à-dire d'un regard, qui « se traduit par une lecture ternaire des faits et des relations »(Moscovici, 1984, p. 9), oblige, comme nous l'avons déjà souligné dans un précédent article (Haas, Masson, 2006), à s'interroger sur la dynamique de l'interaction sujet - chercheur et de ses répercussions sur l'objet. En poursuivant le travail d'élucidation des positions qu'occupent et s'assignent, réciproquement, les protagonistes de cette interaction singulière, nous nous centrerons ici sur la question de l'interprétation de ce qui est dit, mais, aussi, de ce qui est tu.

\section{L'ENTRETIEN : UNE INTÉRACTION SOCIALE SINGULIÈRE}

Pour caractériser la situation d'entretien et la distinguer d'autres situations de recueil de données, de nombreux auteurs en sciences sociales (Jodelet, 2003 ; Kauffmann, 1996 ; Nils, 2003) ont tendance à rapprocher l'entretien non directif de la conversation, c'est-à-dire, d'une interaction au cours de laquelle les protagonistes impliqués se livrent, sans contraintes, à des productions discursives, relatives à un objet particulier. L'analogie avec la conversation permet de montrer que les contenus recueillis ne sont pas prédéterminés, que chaque interviewé dispose, en principe, de toute latitude pour exprimer et verbaliser sa pensée, d'insister sur l'importance de la quête d'une certaine naturalité et de souligner que, dans cette situation particulière de recueil de données, le chercheur est son propre instrument (Jodelet, 2003). Mais cette analogie masque un fait important : la situation d'entretien est une situation d'interaction sociale particulière, reposant, comme le soulignent Bourdieu, Chamboredon et Passeron, sur « une relation sociale (...) profondément artificielle », qui « rompt la réciprocité des échanges coutumiers » et inciterait, selon les auteurs, « les sujets à produire un artefact verbal »(1968/1983, p. 61-62). Le regard psychosocial, selon Serge Moscovici (1970, 1984), permet, en insistant sur l'interaction sujet alter - objet, d'envisager une autre analyse de la situation d'entretien, que celles d'une conversation ordinaire, au cours de laquelle les sujets révèleraient leur vérité ou, au contraire d'une relation sociale, marquée par la violence symbolique faite à l'interviewé et ne générant que des productions artificielles.

Il s'agit, dès lors, de s'interroger véritablement sur la dynamique de la relation interviewé - interviewer et de chercher à saisir qui est l'autre pour l'interviewé, mais, aussi, qui est l'autre pour l'interviewer, c'est-à-dire de procéder à l'analyse des positions et des statuts réciproques qu'occupent, mais aussi que s'octroient et s'assignent

* CRPCC, EA 1285, Université européenne de Bretagne, Université de Bretagne occidentale, CS 93837, 20 rue Duquesne, 29238 cedex 3.

<estelle.masson@univ-brest.fr>

** Université Lumière Lyon 2, Institut de psychologie, GRePS (Équipe PSeCO), 5 avenue Pierre MendèsFrance, 69676 Bron cedex.

<valerie.haas@univ-lyon2.fr> 
mutuellement, les protagonistes de la situation, dans leur rapport avec l'objet.

\section{QUI EST L'AUTRE QU'INTERROGE L'INTERVIEWER ?}

Dans le jeu à trois pôles qu'est la situation d'entretien, il nous semble intéressant de commencer par nous pencher sur celui de l'interviewer. Qui est, pour le chercheur, cet autre, qu'il a convié à s'engager dans un entretien, en qualité de quoi l'interroge-t-il ? Cette première question est plus vaste et plus complexe qu'elle ne peut paraître ; elle ouvre la question cruciale de l'épistémè du sujet en psychologie sociale.

Dans la présentation du numéro spécial «Nouvelles voies en psychologie sociale» du Bulletin de psychologie, Serge Moscovici constatait, avec regrets, que la psychologie sociale avait « acquis la respectabilité (...) au prix d'une désocialisation du concept même d'homme dont elle avait pour devoir d'élaborer la psychologie » (Moscovici, 1992, p. 137). Les termes employés, pour désigner l'individu, constituent un bon indicateur de cette désocialisation : «Les psychologues américains parlent de scientifique "naïf", "intuitif" ou "profane". Or cette dénomination et cette conception très répandues nous paraissent inadéquates. (...) en considérant l'homme comme un scientifique "naïf", on en fait une sorte d'Adam, au jour de sa création, dépourvu de préjugés, de schèmes des choses, un individu présocial ouvrant des yeux ingénus sur un monde de pures impressions sensorielles qui n'ont pas encore été coordonnées en une structure conceptuelle, d'un genre ou d'un autre » (Moscovici, Hewstone, 1984, p. 546). Or, comme le souligne Moscovici, dans la vie sociale, dans la vie mentale, «tout est nécessairement social par nature » (Moscovici, 1986, p. 62), et ce n'est pas «en nous divisant en "naïfs" et "savants", hommes de la rue et hommes de cabinet» (p. 62), que nous parviendrons à comprendre les phénomènes sociaux. Il rappelle, d'un autre côté, que « la psychologie sociale, telle qu'elle a été conçue à l'origine, enjoignait à la psychologie d'unir ses forces à celles de l'anthropologie, de la sociologie et de la linguistique en vue d'étudier une classe particulière de phénomènes qui leurs étaient communs. Il s'agit, et cela ne surprendra personne, de la pensée, du sens commun et de la communication dans une culture déterminée » (Moscovici, 1992, p. 137). Dans une telle perspective, le sujet n'est plus un naïf, mais un être social, appartenant à une culture donnée et dont les savoirs sont réhabilités. Il est expert de ce vaste domaine de connaissances, appelé sens commun, dont il détient, en tant qu'individu particulier, non pas tous les savoirs, mais un nombre largement suffisant pour rendre le monde distinct et signifiant. Lorsque nous, chercheurs, travaillant dans le cadre théorique des représentations sociales, demandons à un individu de nous accorder un entretien, c'est bien à un tel sujet que nous nous adressons.

Pour saisir comment les représentations sociales participent et procèdent de la culture, pour isoler leurs contenus et voir comment ils se combinent et se structurent, pour comprendre comment les sujets donnent sens à leurs pratiques et croyances, nous nous appuyons «sur ce qu'ils disent qu'ils font, et sur ce qu'ils disent des raisons qui les ont poussés à faire ce qu'ils ont fait » et sur «ce que les gens disent de ce que les autres font et des raisons qui les y ont poussés » (Bruner, 1990, p. 31). Car, comme le souligne Bruner, «une psychologie sensible à la dimension culturelle (...) par-dessus tout, (...) doit s'intéresser à ce que les gens disent du monde où ils vivent »(Bruner, 1990, p. 31); c'est là, selon lui, que peuvent être saisies les significations et les interprétations, que produisent les individus.

Pour souligner le caractère « non naïf », que nous reconnaissons aux sujets que nous interviewons, le terme " informateur », utilisé par les anthropologues, pourrait sembler plus pertinent (Masson, 2001). Cependant, cette désignation présente un double inconvénient. D'une part, elle suggère l'existence d'un savoir déjà constitué, d'un savoir, pour ainsi dire unique, figé et intemporel, dont serait détenteur l'interviewé et tend, d'autre part, à réduire la tâche de l'interviewer au recueil le plus précautionneux possible de ce savoir et occulterait, dès lors, un aspect important de la dynamique de l'entretien. En effet, la situation d'entretien non directif est une situation sociale de type «face à face », temporellement et spatialement localisée. C'est un « ici et maintenant », où se déploient les processus d'influence réciproque. Nous y reviendrons.

\section{L'ALTÉRITÉ DE L'INTERVIEWER POUR L'INTERVIEWÉ}

Devereux remarque que l'on s'interroge habituellement peu sur l'influence des caractéristiques du savant, et émet l'hypothèse que tel est le cas, « peutêtre parce qu'on pense que la science est supra personnelle? » (1967/1980, p. 193). Pourtant, il est difficile de faire l'économie de ce questionnement, lorsqu'il s' agit d'entretien. En effet, le chercheur est une condition nécessaire à la situation, c'est lui, qui initie l'interaction au cours de laquelle les matériaux discursifs, relatifs à l'objet, sont élaborés et, conformément au schéma ternaire de Moscovici, on sait que la relation d'Égo (l'interviewé) à l'Objet est médiatisée par l'Alter (l'interviewer). Mais quel 
type d'alter incarne l'interviewer pour l'interviewé ? S'agit-il d'un alter strict ou d'un alter ego ? Qui est cet autrui qu'est le chercheur, pour le sujet, et dans quelle mesure la représentation, que se fait le sujet de cet autrui, détermine-t-elle les contenus recueillis lors d'entretiens?

La recherche réalisée, dans le Bocage, par Favret-Saada sur la sorcellerie (1977), apporte un éclairage intéressant sur ces questions, même si la méthodologie utilisée est l'observation participante et non, à proprement parler, l'entretien non-directif. Elle révèle, cependant, plusieurs aspects importants, relatifs à la perception du chercheur par le sujet, et de ses répercutions sur ce qui est verbalisé à propos de l'objet. Au fil de sa recherche, FavretSaada occupe différentes positions : une position extérieure à l'univers référentiel de la sorcellerie (celle de l'ethnologue, en quête d'informateurs), puis, successivement, deux positions au sein de cet univers, qui ne fait sens que pour celui qui «y est pris » (celle de « désorceleuse » et celle «d'ensorcelée »). Suivant les positions qu'elle occupera et, plus précisément, suivant les positions, que lui assigneront ses interlocuteurs, elle accèdera à des discours et des contenus spécifiques. Lorsque «le paysan parle à l'autre, au savant, il s'objective et ne dit rien »(Favret-Saada, 1977, p. 36), si ce ne sont des balivernes, destinées à convaincre l'ethnographe qu'il « est aussi doué qu'elle pour se distancier d'un "objet" nommé sorcellerie, [marquant] ainsi son droit éminent à participer du même système symbolique que le savant » (Favret-Saada, 1977, p. 36-37). Ce n'est qu'en suite d'une méprise (un couple de paysans, les Badin, qui reconnaîtront dans un premier temps en elle une désorceleuse, puis une ensorcelée), qu'elle parviendra à pénétrer l'univers de la sorcellerie. Ainsi, comme le souligne Favret-Saada, «le premier point à élucider, quand on fait de l'ethnographie des sorts, c'est donc de savoir à qui chaque "informateur" croit s'adresser, puisqu'il tient des discours si radicalement différents, selon la place où il situe son interlocuteur » (Favret-Saada, 1977, p. 36).

Même lorsque l'objet de recherche est moins spécifique que la sorcellerie, où l'on ne parle pas de l'objet, car «la sorcellerie, c'est de la parole, mais une parole qui est pouvoir et non savoir ou information »(Favret-Saada, 1977, p. 26), il est essentiel que le chercheur s'interroge sur la position que lui assigne l'interviewé, car c'est en fonction d'elle que le sujet se situera lui-même dans des espaces référentiels spécifiques et élaborera des discours particuliers ne faisant véritablement sens, que par rapport à eux ${ }^{1}$.

1. Il sera, donc, nécessaire de s'y référer au moment de l'analyse et de l'interprétation des contenus recueillis.
L'interviewé ne s'adresse jamais à un interviewer abstrait, mais, toujours, à un individu singulier et la façon dont se présentera l'interviewer, tout autant que ses caractéristiques personnelles (sexe, âge, mais aussi apparence physique, style, etc.) et son rapport suggéré à l'objet, constituent autant d'éléments qu'utilisera l'interviewé, pour définir la position supposée de l'interviewer, position en regard de laquelle il adoptera lui-même une position particulière.

Face à un alter strict, un chercheur perçu comme expert d'un objet scientifique, il sera, bien souvent, tenté d'affirmer son ignorance: «vous savez mieux que moi », de lui suggérer d'interroger quelqu'un d'autre, quelqu'un de plus compétent, comme le fait cette mère de famille, lors d'une recherche portant sur les représentations de l'alimentation (Masson, Moscovici, 1997): «il faudrait demander à un spécialiste, moi je ne sais pas... ou même j'ai une amie... elle s'est toujours intéressée à la diététique, enfin à ces choses là, faudrait lui demander à elle... moi je sais pas... c'est un cuisinier qu'il faudrait interroger... moi je peux pas dire » ou, encore, de se borner à la restitution de discours dominants sur la question : «tout le monde sait que le plus important est d'avoir une alimentation équilibrée et adaptée à son âge ». Mais qui a pratiqué l'entretien non directif comme une maïeutique, sait qu'au fil de l'entretien le statut de l'interviewer évolue. Son écoute attentive et bienveillante, l'empathie, dont il fait preuve, permettent, peu à peu, l'établissement d'une relation de confiance. Il cesse alors d'être perçu comme cet étranger, qui interroge et questionne et devient progressivement l'accompagnateur du travail d'auto-analyse et d'explicitation (Bourdieu, 1993, p. 914) auquel se livre l'interviewé.

De cet accompagnement dans une élaboration originale (l'interviewé ne s'étant, le plus souvent, jamais véritablement interrogé sur l'objet, avant l'entretien), émerge parfois une complicité, qui transmue la perception de l'interviewer d'un alter strict en quelqu'un de proche : « je vous en raconte des choses, hein... on dirait qu' on se connaît depuis longtemps », dont l'aptitude à comprendre ce qui est dit est implicitement postulé.

Dans une recherche récente, consacrée aux conséquences de l'esclavage sur les mémoires et identités sociales des réunionnais, Gaëlle Deschamps (2006) s'interroge sur sa place dans cette étude, en tant que métropolitaine (tous les entretiens ont été menés en métropole). Elle évoque la relation de confiance qui se noue, au fur et à mesure de l'interaction. Un sujet déclarant, dès le départ de l'entretien : « j'ai jamais eu l'audace de parler avec un zorey [métropolitain] », puis, plus tard, «même vous, j'avais peur de vous parler comme ça». Dans le même ordre d'idée, 
Deschamps cite le fait que des interviewés se mettaient spontanément, en cours d'entretien, à lui parler créole, "alors qu'ils ne pouvaient pas $a$ priori savoir qu'elle comprenait cette langue ». Ce que montre la pratique, c'est que, dès la relation de confiance est établie, l'interviewé ne s'adresse plus à un autre unique (Haas, Masson, 2006), mais, bien souvent, il assigne et fait incarner provisoirement, à l'interviewer, les différentes positions qu'il mobilise et occupe, parfois, temporairement lui-même, pour expliciter sa penser.

Parallèlement, le passage par le regard ternaire (Serge Moscovici, 1970 ; 1984) se révèle être une grille de lecture pertinente et utile pour envisager, analyser, voire interpréter, à travers le type d'interaction (interviewer-interviewé(e)-objet) spécifique à l'entretien de recherche, un moment délicat et encore peu pris en compte dans notre domaine d'étude : le silence.

En effet, celui-ci occupe souvent une place importante dans la relation qui se noue entre l'interviewer et l'interviewé, mais il est très peu pris en compte dans les manuels consacrés à cette technique, dans les enseignements faits aux étudiants, comme dans les analyses et interprétations avancées dans nos travaux de recherche. L'équilibre difficile réside, parfois, dans le fait de (ne pas) transformer ce qui n'aurait pas été dit ${ }^{2}$ ou de mal interpréter ce qui n'aurait pas été échangé. Or, le silence peut être considéré comme une forme de communication adressée à l'autre, au chercheur, que celui-ci a pour tâche d'explorer, au même titre que le contenu du discours et qui se révèle, parfois, être tout à fait révélateur, symptomatique de l'objet étudié, comme nous allons tenter de le démontrer.

\section{APPRIVOISER LE SILENCE AU COURS DE L'ÉCHANGE}

Dans le cadre de nos enseignements, une bonne partie de l'apprentissage de la pratique d'entretien réside dans cette "gestion du silence», que nous nous devons de transmettre aux étudiants. En effet, ces derniers, dès les premiers entretiens effectués sur le terrain, nous disent souvent être « paniqués », « mal à l'aise », lorsqu'ils découvrent avec stupeur que l'interviewé peut, aussi, à certains moments de l'entretien, «ne rien vouloir dire». À ce stade de l'apprentissage, ils n'ont de cesse de relancer rapidement l'entretien, avec des questions souvent dénuées de tout intérêt pour l'enquête : ils comblent la hantise des silences par du discours.

2. Faire parler les silences, en leur donnant le sens attendu des hypothèses ou entendre les réponses désirées à nos propres interrogations.
Ainsi, apprendre la technique d'entretien, c'est donc aussi savoir «apprivoiser» cet instant, ce moment tant redouté, qui est souvent une respiration nécessaire dans l'interaction et constitue, parfois, le squelette, la matière première, sur laquelle le chercheur fondera une part essentielle de son interprétation par la suite ${ }^{3}$.

\section{UN SILENCE QUI EN DIT LONG...}

Un retour sur différentes références bibliographiques, nous conduit à réfléchir à la place qui est faite au silence, dans les manuels de sciences sociales consacrés à l'entretien. Que dit la littérature à ce propos et quelle place est donnée dans ce cadre à la relation interviewer/interviewé(e) ? Ce silence qui se noue, se crée dans l'entretien, est envisagé de manière très disparate dans les ouvrages consacrés aux méthodes d'enquête. Interviewers et interviewés y sont envisagés toujours alternativement, mais jamais conjointement.

Ainsi, pour la psychologie sociale, du côté de l'interviewé(e), le silence est perçu d'une part, comme «un mécanisme de défense », offrant une interprétation clinique de la situation (Pinto, Grawitz, 1964 ; McAdams, 1998) ou, au contraire, comme un trouble du langage, apparenté au « débit verbal, aux hésitations, aux pauses ou tremblements de la voix » (Nils, Rimé, 2003, p. 169). Paradoxalement, le silence de l'interviewer est envisagé d'une tout autre manière. Blanchet, par exemple, présente, dans plusieurs de ses ouvrages et articles (1982, 1983, 1985, 1991, 1992), des résultats de travaux où il étudie les effets de différents types d'intervention sur le contenu et la forme du discours de l'interviewé. Dans le cadre de ce type d'expérimentation, l'auteur mentionne, par exemple, la technique du stress interview, qui vise à repérer l'effet des silences, amplifiés artificiellement, de l'interviewer «non directif » sur l'interviewé (Blanchet, 1985, p. 65). Ici, les effets sont apparentés à une forme de prise de pouvoir sur l'enquêté(e), à une volonté de domination, largement relayée par une vision dissymétrique de l'entretien, où l'interviewer a toujours le dessus.

La majorité de ces études, laisse entrevoir qu'en réalité, il est toujours question de l'interviewé à travers ces axes d'analyse. Ainsi, on étudie soit les effets du silence de l'interviewer sur l'interviewé soit les causes ou raisons du silence de l'interviewé. Il n'est jamais fait mention ni de l'interaction, qui se joue dans le cadre de l'entretien entre

3. Dans le cadre de la retranscription des entretiens, les étudiants apprennent à noter les silences avec leur durée, matière essentielle à l'analyse de contenu qui suivra. 
les deux protagonistes, ni même des effets et implications de ce silence sur le chercheur lui-même. Cette grille de lecture offre une vision binaire de l'interaction interviewer-interviewé(e), à sens unique, que l'on pourrait même relier au schéma behavioriste, tant le silence est apparenté à un stimulus dont on observerait les causes ou effets directs.

Dans les sciences sociales, plus généralement, une bonne partie de la littérature (qui reste, cela dit, encore mineure à ce propos) est consacrée à la question des non-réponses, considérée comme « une part de silence en soi », imposée au chercheur : silence sur les questions posées et, de fait, silence sur les données. Cet aspect est développé, de manière relativement indifférenciée, selon les techniques utilisées : que l'on parle du questionnaire ou de l'entretien proprement dit, les «non réponses » sont, plus ou moins, appréhendées de la même manière.

Déjà, en 1982, François de Singly faisait le point à ce propos, dans un article très complet portant sur la «gestion sociale des silences». Le silence est clairement associé, pour cet auteur, à une «nonréponse », puisque seule cette modalité est étudiée sur l'ensemble de l'article, malgré un titre clairement évocateur. Ces «sortes de silence» sont, souvent, apparentées à une «anomalie » intrinsèque au questionnaire ou, encore, à une incompétence du sujet sur certaines thématiques, empêchant, de fait, le chercheur de pouvoir en dire plus sur ses données. Singly explique que l'on peut, aussi, analyser ces non-réponses comme une intériorisation de la domination sociale, une forme de « silence volontaire ${ }^{4}$, type d'interprétation très récurrent en sociologie et à propos duquel les études ne manquent pas. Cela dit, il faut, aussi, pouvoir dépasser cette question de la domination, car l'on peut également «se taire non pas parce que l'on ne sait pas ou qu'on croit ne pas savoir mais parce que l'on ne veut pas dire » (Singly, 1982, p. 38).

Certains silences pourraient, donc, révéler une résistance à la divulgation de pensées, de comportements, de savoirs privés, qui doivent le rester. Singly parle, ainsi, du silence, dans l'enquête sociologique, apparenté à la gestion d'un secret, en référence à Goffman, c'est-à-dire, du droit de Soi au secret : « Refuser de répondre peut s'avérer la seule parade par laquelle le sujet interrogé pourra se

4. «Les silences volontaires traduiraient l'intériorisation d'une domination sociale qui chasse du terrain politique les femmes davantage que les hommes, les ouvriers davantage que les cadres supérieurs, les personnes d'un niveau scolaire primaire davantage que les personnes d'un niveau scolaire secondaire ou universitaire » (De Singly, 1982, p. 38). protéger, en retenant les éléments de réponse qui risqueraient de lui "faire perdre la face" vis-à-vis des autres, mais aussi de lui-même » (Singly, 1982, p. 48). C'est cet autre qu'il nous faut probablement interroger dans l'entretien, comme une présence non anodine du chercheur, marquant les sélections, les silences ou «oublis volontaires» que fera le sujet dans son discours.

Du côté de la psychologie sociale, un recueil d'article sur cette thématique (Bauer, Joffe, 1996 ; Jodelet, 1996 ; Turner, Michael, 1996) fait le point sur la question. Les auteurs regrettent le peu de cas qui est fait de cet aspect, pourtant crucial, de l'enquête, quand les sujets choisissent de «dire qu'ils ne savent pas » (en référence à la notion d'ignorance déclarée) ou choisissent, tout bonnement, de se taire. Les psychosociologues utilisent, ici, des axes d'analyses assez similaires à ceux de la sociologie et s'intéressent, en particulier, à des grands thèmes d'études assez propices à ce type de comportement: la science (Bauer, 1996), les affaires publiques et la politique (Joffe, Farr, 1996) ou encore les comportements sexuels (Giami, 1996).

Ce qui est frappant dans les travaux qui sont actuellement consacrés aux silences ou aux non réponses des interviewés, des enquêtés, c'est que «ces retenues» dans le discours sont rarement envisagées comme une «adresse » faite à l'interviewer, pour ce qu'il est ou représente, dans le contexte spécifique de l'interaction. Dans les manuels traitant de la technique d'entretien ou, plus généralement, de l'enquête, l'interviewer apparaît peu comme la raison du silence, l'objet d'étude encore moins. Chaque pièce du puzzle de l'entretien semble totalement indépendante l'une de l'autre. D'où l'intérêt de revenir à la triangulation proposée par Moscovici.

\section{LE REGARD TERNAIRE COMME MANIËRE D'APPRÉHENDER LE SILENCE DANS L'ENTRETIEN}

En effet, en écho à la première partie de cet article, nous pourrions, d'emblée, considérer que la présence (physique, imaginaire, symbolique) de l'interviewer occupe une place centrale dans ce qui est dit ou dans ce qui est tu par le sujet interviewé. En outre, celle de son magnétophone (qui enregistre les réponses et, par là même, conserve les traces du discours) n'est absolument pas anodine. À l'extrême, Singly considère même que ce sont là des formes de violence symbolique, qui sont adressées à la personne interrogée, véritable «offense territoriale», au sens de Goffman (1973) : «La non-réponse enrichit les procédés d'évitement et de protection que recensent les codes de politesse en y ajoutant la résistance par le 
silence puisque l'empiétement ou l'offense qu'exercent enquêtes et sondages sont par euxmêmes étrangers à tout code de civilité quotidienne » (Singly, 1982, p. 62). Parallèlement, nous pouvons aussi pointer du doigt le fait que, dans l'entretien, l'interviewé sait, aussi, parfaitement, que les données, qu'il fournit sont destinées à être publiques (bien que l'on tente de lui prouver le contraire, en lui expliquant, par avance, le principe de l'anonymat!). Toutes ces raisons, au moins, peuvent nous conduire à penser que l'interviewé(e) peut - à certains moments de l'entretien - préférer faire silence, se taire, au regard du monde extérieur, symbolisé ici par le chercheur.

En effet, le silence peut-être fait sur certains thèmes, par crainte, honte ou pudeur et touche, parfois, à une certaine forme d' "interdiction du groupe », sorte de tabou (Haas, 2002a, 2002b). Cette forme d'interdit pourrait être ici entendue et repensée par la place que prend le chercheur, venant « re-présenter» la normalité socialement attendue, le «monde » extérieur. Il pourrait être entendu comme un écho à la "désirabilité sociale » 5 , où la parole «retenue » s'inscrirait comme une méfiance à l'encontre de l'interviewer.

Pour illustrer nos propos, prenons un exemple, choisi dans une recherche récente (Jacquart, Haas, 2006), durant laquelle nous avons interviewé des personnes, victimes d'inondations dans le Nord de la France. Cette catastrophe naturelle, "inimaginable et exceptionnelle » a, très vite, été interprétée dans le discours local comme « un coup monté par les Parisiens, qui auraient détourné les eaux surabondantes de la Seine, pour qu'elles viennent se déverser dans la Somme déjà au seuil critique » ${ }^{6}$. Cependant, durant les cinquante entretiens menés avec les habitants et quel que soit leur degré de proximité de l'événement, les sujets ne faisaient jamais mention, directement, de ce que l'on peut clairement (de l'extérieur) apparenter à une rumeur. En fait, ce silence n'est pas étonnant et Festinger abordait cet aspect particulier de la rumeur, qu'il associait à une forme «d'activité secrète » (Back, Festinger et coll. 1950, p. 123). Elle tient, principalement, au fait que, si l'on adhère à l'explication fournie par la dite rumeur, elle n'en a justement pas le statut. Cela tient, aussi, à la connotation

5. Cette dernière qui consiste, pour le sujet, à vouloir fréquemment, dans les entretiens en particulier et dans les enquêtes en général, donner une image de soi favorable, en s'attribuant des caractéristiques, des idées ou valeurs socialement positives.

6. Ce type d'interprétation, venant faire sens dans la mémoire collective de cette population, la Picardie ayant été, notamment au cours des deux dernières guerres mondiales, un champ de bataille, sorte de bouclier préservant, bien souvent, les Parisiens des avancées ennemies. péjorative de ce terme. Consistant à en faire une information erronée, «irrationnelle » et absurde, elle ne peut s'appliquer à ce que les sujets considèrent comme avéré et valide. Mais plus encore, dans le cadre de ces échanges, nous n'étions pas reconnues comme des personnes de confiance, comme des « alter ego », pour reprendre encore les termes de Moscovici. Une méfiance s'installait d'emblée à notre égard car, à l'époque où nous menions les entretiens, bon nombre de quotidiens nationaux se raillaient de cette «bonne plaisanterie » des Picards. Les colonnes de la presse nationale tentaient de «raisonner» ces habitants « naïfs », « profanes », en leur offrant une explication rationnelle, scientifique et objective... Les habitants n'étaient donc pas prêts à nous livrer, « en l'état », le fond de leur pensée. Ce n'est que grâce à une bonne connaissance du terrain et à l'application d'une triangulation méthodologique, mise en place sur l'ensemble de l'étude ${ }^{7}$, que nous avons pu dépasser ce silence et l'interpréter comme une donnée à part entière de la recherche.

Le but du recueil verbal n'est-il pas, alors, d'appréhender ce que les sujets nous disent, mais aussi ce qu'ils taisent, ce qu'ils cherchent à mettre de côté, à effacer, voire à dissimuler et, surtout, à tenter d'en comprendre les raisons ? D'où la nécessité de s'intéresser, plus avant et de manière réflexive, à cette position que nous occupons, en tant que chercheur, dans la situation de recueil verbal.

Ainsi, ce que nous pourrions nommer «silencesensible » dans les entretiens pourrait être caractérisé par ce qui est tu volontairement par le sujet, au cours de l'interaction. Se taire serait donc une forme de silence adressé au chercheur, face à un objet qui semble problématique dans l'ici et maintenant de la relation. Silence que le chercheur aura pour tâche de déchiffrer.

Rejoignant nos propos, Le Breton, consacrant un ouvrage entier à la thématique du silence, fait une distinction éclairante. En effet, la langue grecque révèle que l'on peut distinguer sigân (être en silence) et siôpân (se taire). Le premier verbe renverrait à la possibilité, pour un individu, « d'être silencieux », comme, par exemple, pour « reprendre son souffle », " réfléchir », formes de respiration, inhérente à la situation d'échange, que l'on pourrait rapprocher d'un comportement non verbal. Alors que le fait de «se taire» est un acte volontaire et adressé à l'autre : «Dans le fait de se taire il y a comme un retranchement hors du

7. Au cours de cette recherche, nous avons utilisé plusieurs outils méthodologiques : une recherche documentaire, des sous-groupes ou, encore, le recueil d'une analyse de presse importante. 
langage, une volonté de ne plus donner sa parole et de le faire sentir à l'autre. (...) Dans le cadre d'un échange, l'un des protagonistes garde le silence et projette ainsi une signification directe susceptible d'interroger les autres »(Le Breton, 1997, p. 26). Le fait de se taire viendrait marquer une réticence à livrer quelque chose, que le chercheur ne doit pas savoir sur l'objet, en partie pour la place qu'il prend dans l'interaction, où il est souvent pris, dans ces cas précis, pour un «alter strict», un autre extérieur. Ce type spécifique de «silence choisi » est, bien sûr, à distinguer de l'impossibilité de dire, de l'indicible ou de l'intransmissible, caractéristique, lui aussi, de certaines situations d'entretien ${ }^{8}$. Ainsi, «je ne peux pas vous dire», qui témoigne d'une impossibilité de parole, adressée au chercheur, est bien différente de « je ne veux pas vous dire », pour le cas qui nous occupe.

\section{CONCLUSION : DE L'INTERPRÉTATION DE CE QUI EST DIT ET DE CE QUI EST TU}

Ainsi, comme l'écrit Olivier de Sardan (1995), il n'est pas question d'arriver à « une version véridique » du discours. À ce propos, Kaufmann avance l'idée de «fable de vie », qui caractérise la manière dont le chercheur peut dépasser les silences et les non-dits, « en écoutant attentivement les fables qu' on lui raconte, car c'est à travers elles qu'il trouvera des indices » (Kaufmann, 1996, p. 69). La quête de ces indices peut être, en partie, effectuée en utilisant la technique de triangulation des méthodes (Haas, Kalampalikis, 2010), comme nous l'avons déjà indiqué, qui offre au chercheur «la possibilité de combiner plusieurs

8. On peut penser, par exemple, à des entretiens menés avec des rescapés des camps de la mort, tels des rescapés de génocides, qui sont particulièrement difficiles à mener, tant le fait de parler sur l'événement pose la douloureuse question de mettre en mots un événement traumatique. méthodologies dans l'étude d'un même phénomène » (Denzin, 1978, p. 291). Certains outils méthodologiques (de type projectifs, par exemple), pouvant aussi intervenir, non pas comme outil de substitution, mais comme complément à l'entretien (Haas, 2004).

Mais surtout, au moment de l'analyse des entretiens et en vue de leur interprétation, le chercheur pourra réinjecter le cadre d'analyse du regard ternaire selon Moscovici. La réflexion induite, à ce propos, devrait permettre de (re)-considérer la place que chacun a pris dans la relation, mais aussi d'effectuer un retour réflexif sur notre propre place de chercheur, dans le présent de la relation (étant tantôt perçu par le sujet comme un alter strict, tantôt comme un alter ego). Ce regard, porté sur le contenu de l'entretien, sur ce qui a été dit, comme sur ce qui a été tu, devrait permettre de s'interroger, tant sur la dynamique de l'interaction qui s'est tenue, que sur ses effets sur l'objet étudié. D'ailleurs, comme le souligne Devereux, « l'observation d'un sujet par un observateur a toujours comme complément, la contre observation de l'observateur par le sujet» (1967/1980, p. 375). L'interviewé parle à quelqu'un, il construit et adresse sa production narrative à une personne en particulier. Ce que recueille l'interviewer, dès lors, ne saurait être considéré comme la vérité du sujet, une vérité permanente et intemporelle; ce qui est dit, fait véritablement sens pour celui qui l'énonce dans l' «ici et maintenant » de l'interaction interviewé/interviewer et rien ne permet d'étendre la pertinence du propos à tous les autres contextes. Il est, donc, important de tenir compte des conditions d'actualisation du discours et de reconnaître, aux matériaux recueillis, leur caractère non immuable, c'est-à-dire accepter qu'ailleurs, avec quelqu'un d'autre, dans d'autres conditions, le sujet puisse penser autrement, formuler un discours différent, qu'une autre vérité soit plus signifiante pour lui « sujet».

\section{RÉFÉRENCES}

Back (Kurt), Festinger (Leon), Hymovitch (Bernard), Kelley (Harold H.), Schachter (Stanley), ThiBaut (John).- A method of studying rumor transmission, Theory and experiment in social communication, Research center for dynamics institute for social research, Michigan, MI, 1950, p. 118-123.

BAuER (Martin W.), Joffe (Hélène).- Meanings of self-attributed ignorance : an introduction to the symposium, dans Symposium on «self-attributed ignorance »,
Social science information / Information sur les sciences sociales, 35, 1, 1996, p. 5-13.

BAUER (Martin W.).- Socio-demographic correlates of DK-responses in knowledge suveys : self-attributed ignorance of science, dans Symposium on «self-attributed ignorance », Social scienceinformation / Information sur les sciences sociales, 35, 1, 1996, p. 39-68.

Blanchet (Alain).- Dire et faire dire. L'entretien, Paris, Armand Colin, 1991. 
BLANCHET (Alain).- Épistémologie critique de l'entretien d'enquête de style non directif. Ses éventuelles distorsions dans le champ des sciences humaines, Bulletin de psychologie, 36, 1-4, 358, 1982, p. 187-194.

BlAnCHET (Alain).- L'entretien, à l'interface du psychologique et du social, Bulletin de psychologie, 36, 11-15, 360, 1983, p. 565-570.

Blanchet (Alain).- L'enquête et ses méthodes: l'entretien, Paris, Nathan, 1992.

BLANCHET (Alain).- L'entretien dans les sciences sociales. L'écoute, la parole et le sens, Paris, Dunod, 1985.

Bourdieu (Pierre), ChAmboredon (Jean-Claude), PAsseron (Jean-Claude).- Le métier de sociologue [1968], Paris, Mouton, 1983.

Bourdieu (Pierre).- Comprendre, dans Bourdieu (P.), La misère du monde, Paris, Seuil, 1993, p. 903-939.

BRUNER (Jerome).- ... car la culture donne forme à l'esprit: de la révolution cognitive à la psychologie culturelle, Paris, Eshel, 1990.

DENZIN (Norman K.).- The research act, Chicago, Aldine, 1978.

Deschamps (Gaëlle).- Mémoires sociales de 1'esclavage : l'exemple réunionnais, mémoire de Master 2 en psychologie sociale, Université Lumière Lyon 2, 2006.

DEVEREUX (Georges).- From anxiety to method in the behavioral sciences [1967], trad.. fr. De l'angoisse à la méthode dans les sciences du comportement, Paris, Mouton, 1980.

FAVRET-SAADA (Jeanne).- Les mots, la mort, les sorts, Paris, Gallimard, 1977.

Giami (Alain).- Partial non-response and « don't know » responses in surveys on sexual behavior, dans Symposium on «self-attributed ignorance», Social science information / Information sur les sciences sociales, 35, 1, 1996, p. 93-110.

GoFFMAN (Erving).- Les relations en public, Paris, Édition de minuit, 1973.

HaAs (Valérie), Kalampalikis (Nikos).- Triangulation méthodologique à partir de l'énigme du don de sperme, dans Masson (E.), Michel-Guillou (E.), Le cabinet de curiosités : les différentes facettes de l'objet en psychologie sociale, Paris, L'harmattan, 2010, p. 61-76.

HAAS (Valérie), MASsON (Estelle).- La relation à l'autre comme condition à l'entretien, Les cahiers internationaux de psychologie sociale, 71, 2006, p. 77-88.

HaAs (Valérie).- Approche psychosociale d'une reconstruction historique. Le cas vichyssois, Les cahiers internationaux de psychologie sociale, 53, 2002a, p. 32-45.

HAAs (Valérie).- La face cachée d'une ville, dans Ferenczi (T.), Devoir de mémoire, droit à l'oubli ?, Paris, Complexe, 2002b, p. 59-71.

HaAs (Valérie).- Les cartes cognitives : un outil pour étudier la ville sous ses dimensions socio-historiques et affectives, Bulletin de psychologie, 57, 6, 474, 2004, p. 621-633.

HaAs (Valérie).- Les savoirs du quotidien. Transmissions, appropriations, représentations, Rennes, Presses universitaires de Rennes, 2006.

JACQUART (Cécile), HAAS (Valérie).- La rumeur comme modalité de la pensée sociale, dans Haas (V.), Les savoirs du quotidien. Transmissions, appropriations, représentations, Rennes, Presses universitaires de Rennes, 2006, p. 51-67.

Jodelet (Denise).- Aperçus sur les méthodologies qualitatives, dans Moscovici (S.), Buschini (F.), Les méthodes des sciences humaines, Paris, Presses universitaires de France, 2003, p. 139-162.

JODELET (Denise).- Commentary on meanings of selfattributed ignorance, dans Symposium on « self-attributed ignorance », Social science information / Information sur les sciences sociales, 35, 1, 1996, p. 111-120.

JOFFE (Hélène), FARR (Robert).- Self-proclaimed ignorance about public affairs, dans Symposium on « selfattributed ignorance », Social science information / Information sur les sciences sociales, 35, 1, 1996, p. 69-92.

KAUFMANN (Jean-Claude).-L'entretien compréhensif, Paris, Nathan, 1996.

Le Breton (David).- Du silence, Paris, Métailié, 1997.

Masson (Estelle), Moscovici (Serge).- Les mutations dans la pratique alimentaire. Processus symboliques et représentations sociales, Rapport de fin de recherche, Paris, École des hautes études en sciences sociales / Ministère de l'agriculture, 1997.

MAsson (Estelle).- Les formes du manger. Représentations de pratiques alimentaires contemporaines, Thèse de troisième cycle, Paris, École des hautes études en sciences sociales, 2001.

McAdams (Dan P.).- The role of defense in the life story, Journal of personality, 66, 6, 1998, p. 1125-1146.

Moscovici (Serge), Hewstone (Miles).- De la science au sens commun, dans Moscovici (S.), Psychologie sociale, Paris, Presses universitaires de France, 1984, p. 539-566.

Moscovici (Serge).- Introduction. Le domaine de la psychologie sociale, dans Moscovici (S.), Psychologie sociale, Paris, Presses universitaires de France, 1984, p. 5-22.

Moscovici (Serge).- L'ère des représentations sociales, dans Doise (W.), Palmonari (A.), L'étude des représentations sociales, Neuchâtel, Paris, Delachaux et Niestlé, 1986, p. 34-80.

Moscovici (Serge).- Préface, dans Jodelet (D.),Viet (J.), Besnard (P.), La psychologie sociale. Une discipline en mouvement, Paris, La Haye, Mouton, 1970, p. 9-64.

Moscovici (Serge).- Présentation du numéro : nouvelles voies en psychologie sociale, Bulletin de psychologie, 45, 4-7, 405, 1992, p. 137-143.

NiLs (Frédéric), Rimé (Bernard).- L'interview, dans Moscovici (S.), Buschini (F.), Les méthodes des sciences 
humaines, Paris, Presses universitaires de France, 2003, p. 165-186.

Olivier DE SARDAN (Jean-Pierre).- La politique du terrain. Sur la production des données en anthropologie, Enquête, 1, Les terrains de l'enquête, p. 71-112.

PInTo (Roger), Grawitz (Madeleine).- Méthodes des sciences sociales, Paris, Précis Dalloz, Tome II, 1964.
Singly (François de).- La gestion sociale des silences, consommation, Revue de socio-économie, 4, 1982, p. 37-63.

Turner (Jill), Michael (Mike).- What do we know about «don't knows »? Or, contextes of «ignorance », dans Symposium on « self-attributed ignorance », Social science information / Information sur les sciences sociales, 35, 1, 1996, p. 15-38. 